\title{
Article
}

\section{The Effect of Octapeptide Repeats on Prion Folding and Misfolding}

\author{
Kun-Hua Yu ${ }^{\dagger}$, Mei-Yu Huang ${ }^{\dagger}$, Yi-Ru Lee, Yu-Kie Lin, Hau-Ren Chen $(\mathbb{D}$ and Cheng-I Lee *(D) \\ Department of Biomedical Sciences, National Chung Cheng University, 168 University Road, \\ Min-Hsiung Chia-Yi 62102, Taiwan; ykhuna@gmail.com (K.-H.Y.); hb07925@hotmail.com (M.-Y.H.); \\ sane11989@hotmail.com (Y.-R.L.); kie82123@gmail.com (Y.-K.L.); biohrc@ccu.edu.tw (H.-R.C.) \\ * Correspondence: biocil@ccu.edu.tw; Tel.: +886-5-2729157 \\ + These authors contributed equally.
}

check for updates

Citation: Yu, K.-H.; Huang, M.-Y.; Lee, Y.-R.; Lin, Y.-K.; Chen, H.-R.; Lee, C.-I. The Effect of Octapeptide Repeats on Prion Folding and Misfolding. Int. J. Mol. Sci. 2021, 22, 1800. https://doi.org/10.3390/ ijms 22041800

Academic Editor: Jya-Wei Cheng, Shih-Che Sue, Kuen-Phon $\mathrm{Wu}$, Hui-Chun Cheng and Shang-Te Hsu

Received: 31 December 2020

Accepted: 8 February 2021

Published: 11 February 2021

Publisher's Note: MDPI stays neutral with regard to jurisdictional claims in published maps and institutional affiliations.

Copyright: (c) 2021 by the authors. Licensee MDPI, Basel, Switzerland. This article is an open access article distributed under the terms and conditions of the Creative Commons Attribution (CC BY) license (https:/ / creativecommons.org/licenses/by/ $4.0 /)$.

\begin{abstract}
Misfolding of prion protein $(\mathrm{PrP})$ into amyloid aggregates is the central feature of prion diseases. PrP has an amyloidogenic C-terminal domain with three $\alpha$-helices and a flexible tail in the $\mathrm{N}$-terminal domain in which multiple octapeptide repeats are present in most mammals. The role of the octapeptides in prion diseases has previously been underestimated because the octapeptides are not located in the amyloidogenic domain. Correlation between the number of octapeptide repeats and age of onset suggests the critical role of octapeptide repeats in prion diseases. In this study, we have investigated four PrP variants without any octapeptides and with 1, 5 and 8 octapeptide repeats. From the comparison of the protein structure and the thermal stability of these proteins, as well as the characterization of amyloids converted from these PrP variants, we found that octapeptide repeats affect both folding and misfolding of PrP creating amyloid fibrils with distinct structures. Deletion of octapeptides forms fewer twisted fibrils and weakens the cytotoxicity. Insertion of octapeptides enhances the formation of typical silk-like fibrils but it does not increase the cytotoxicity. There might be some threshold effect and increasing the number of peptides beyond a certain limit has no further effect on the cell viability, though the reasons are unclear at this stage. Overall, the results of this study elucidate the molecular mechanism of octapeptides at the onset of prion diseases.
\end{abstract}

Keywords: prion; octapeptide; folding; misfolding; fibril

\section{Introduction}

Transmissible spongiform encephalopathies (TSE or prion diseases) are fatal neurodegenerative disorders with sporadic, infectious and inherited etiologies. The prion proteins $(\mathrm{PrP})$ play an essential role in the pathogenesis of prion diseases. According to the proteinonly hypothesis [1], the normal cellular isoform of the prion protein $\left(\mathrm{PrPC}^{\mathrm{C}}\right)$ misfolds and aggregates into a disease-associated state termed $\mathrm{PrP}^{\mathrm{Sc}}$ (Sc stands for Scrapie) [2]. $\mathrm{PrP}^{\mathrm{C}}$ is sensitive to protease $\mathrm{K}(\mathrm{PK})$-digestion, while $\mathrm{PrP}^{\mathrm{PC}}$ is resistant to $\mathrm{PK}$-digestion. $\mathrm{PrP}^{\mathrm{C}}$ is ubiquitous throughout the central nervous system of mammals [3].

Misfolding of $\mathrm{PrP}^{\mathrm{C}}$ into $\mathrm{PrP}^{\mathrm{Sc}}$ readily causes various types of transmissible spongiform encephalopathies in animals, such as Creutzfeldt-Jakob disease (CJD) in humans, bovine spongiform encephalopathy (BSE) in cattle and Scrapie in sheep. Approximately $15 \%$ of CJD are genetic disorders [4]. Conventional single point mutations and multiple insertion mutations in an octapeptide region in the $\mathrm{N}$-terminal domain contribute approximately equally [5]. Deletion in the octapeptide region of the N-terminal domain occurs in approximately $2 \%$ of the Caucasian population [6].

Nuclear magnetic resonance (NMR) studies indicate that $\operatorname{PrP}^{\mathrm{C}}$ possesses a large $\alpha$ helical domain and a short two-stranded anti-parallel $\beta$-sheet in the C-terminal domain (residues 121-231 inclusive) and a flexible N-terminal domain (residues 23-120 inclusive) [7]. In contrast, $\mathrm{PrPSc}$ is rich with cross $\beta$-sheets, yet few $\alpha$-helical structures [8]. The C-terminal domain of $\operatorname{PrP}$ has been considered an amyloidogenic region because amyloid 
fibrils can be converted from $\operatorname{PrP}_{121-231}$ (equivalent to mouse $\operatorname{PrP}_{23-230}$ ) proteins, which lack the entire unstructured $\mathrm{N}$-terminal domain of the protein [9]. The $\mathrm{N}$-terminal domain of mouse PrP contains five tandem repeats of the octapeptide P(H/Q)GG(-/G/S/T)WGQ sequence including a leading octapeptide PQGGTWGQ from residue 51 to residue 58 (inclusive) and the following four octapeptide repeats PHGG(G/S)WGQ from residue 59 to residue 90 (inclusive). These four octapeptide repeats bind to divalent cation including $\mathrm{Cu}^{2+}, \mathrm{Mn}^{2+}$ or $\mathrm{Zn}^{2+}$ due to the chelating effect of histidine residues. To be noted, the leading peptide in humans is PQGGGTWGQ nonapeptide without histidine to bind $\mathrm{Cu}^{2+}$. Thus, the repeat number of these octapeptides has been considered as four in some studies $[10,11]$.

Across species, the octapeptide region is among the represented portions of the PrP sequence [12], suggesting that this region plays a critical role in $\mathrm{PrP}^{\mathrm{C}}$ function. The octapeptides are structurally flexible and are outside of the amyloidogenic $\mathrm{C}$-terminal domain. This octapeptide region in prion diseases has been greatly underestimated. However, a survey on CJD patients suggested a correlation between the number of octapeptide repeats and the age of onset; when the number of octapeptide repeats is decreased, the onset of CJD is postponed [13], whereas a large number of octapeptide insertions induces large plaques and early onset [14]. In octapeptide deleted PrP, CJD syndrome is not well recognized [4]. In summary, the number of octapeptide repeats is critical in prion diseases and we need to understand how the variation of octapeptide repeats modulates the mechanism of the diseases.

As the number of octapeptide repeats is critical in the pathology of prion diseases, we have worked on full-length mouse PrP carrying 5 repeats of octapeptides (PrP-5oct) without any peptide tag. Its deletion/insertion resulted in three other PrP variants: $\operatorname{PrP}$ without any octapeptide (PrP- $\Delta$ oct), PrP with one (PrP-1oct) and eight octapeptide repeats (PrP-8oct) as illustrated in Figure 1. We have analyzed the protein structure, the structural stability of these protein variants and the kinetics of their amyloid conversion as well as structural characteristics of the amyloid fibrils. The cytotoxicity of these four PrP variants is also examined at the cellular level.

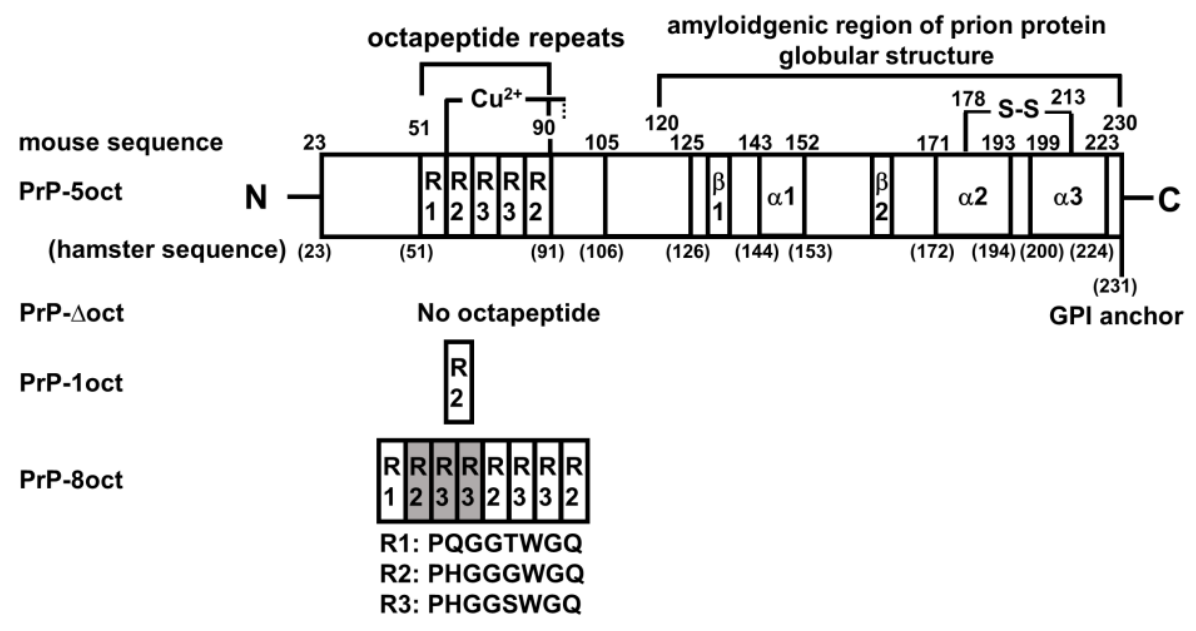

Figure 1. Representative scheme of prion sequence and prion proteins $(\mathrm{PrP})$ variants with different number of octapeptide repeats. The sequences of the octapeptide repeats are represented by R1, R2 and R3. Three $\alpha$-helices are labeled as $\alpha 1, \alpha 2$ and $\alpha 3$. Two $\beta$-strands are labeled as $\beta 1$ and $\beta 2$.

\section{Results}

\subsection{The Number of Octapeptide Repeats Affects the Structure and the Thermal Stability of} Prion Proteins

According to the NMR studies, $\mathrm{PrP}$ is rich with $\alpha$-helices located in the $\mathrm{C}$-terminal domain, while the $\mathrm{N}$-terminal domain is unstructured [7]. Therefore, in the determination of mean residue ellipticity, only the residues in the C-terminal domain (residues 120 to 
230) are counted. As shown in Figure 2a, the circular dichroism (CD) spectra of all PrP variants illustrate high amounts of $\alpha$-helical structures found at negative peaks of $208 \mathrm{~nm}$ and $222 \mathrm{~nm}$. The analysis of the fraction of the secondary structure is shown in the insert of Figure 2a. All PrP variants have the same fraction of $\beta$-sheets. In comparison to PrP-5oct, both deletion and insertion of octapeptides increase the fraction of $\alpha$-helical structure. The observation that the change of $\alpha$-helical structures are related to the number of octapeptides indicates that octapeptides in the N-terminal domain can effectively affect the $\alpha$-helices dominant in the C-terminal domain of PrP. In addition, thermal stability of $\alpha$-helices in $\operatorname{PrP}$ variants measured by their melting temperature $\left(T_{m}\right)$ values is compared in Figure 3 . An increase in $\mathrm{T}_{m}$ was observed in both deletion and insertion of octapeptides. In other words, PrP-5oct has the least thermal stability. The correlation of $\mathrm{T}_{m}$ is very similar to the number of octapeptide repeats vs. the fraction of $\alpha$-helical structure (insert in Figure 2a).
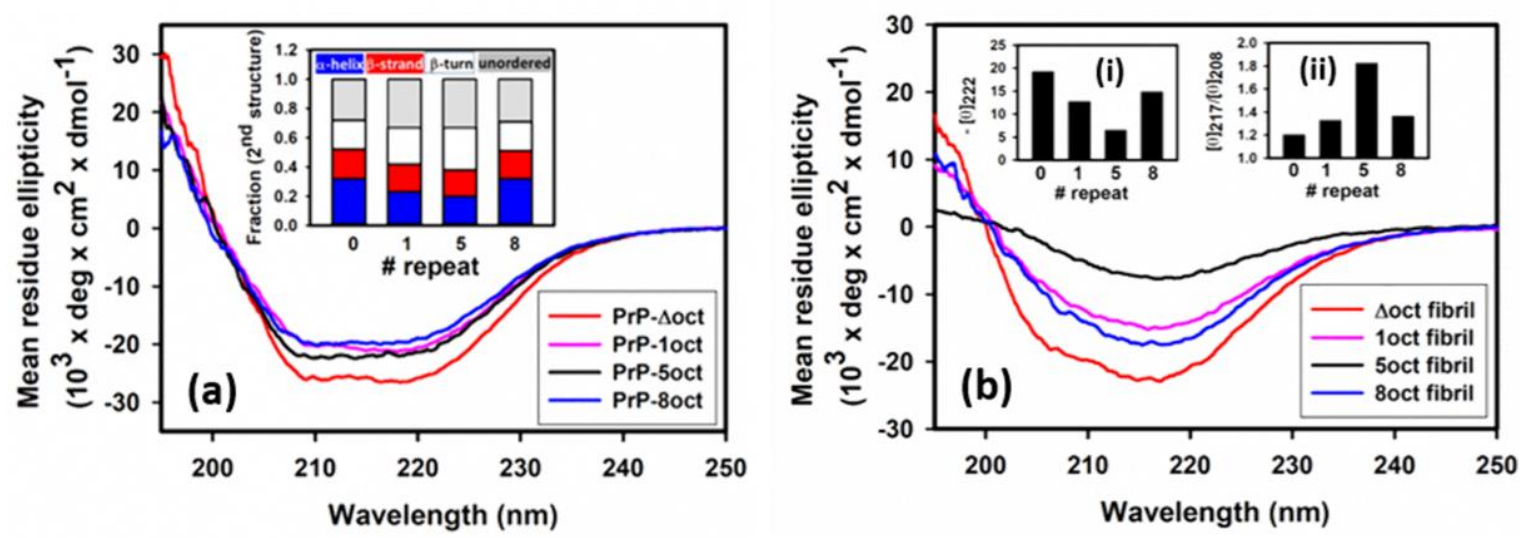

Figure 2. Circular dichroism (CD) analysis of (a) PrP variants and (b) fibrils converted from four PrP variants.

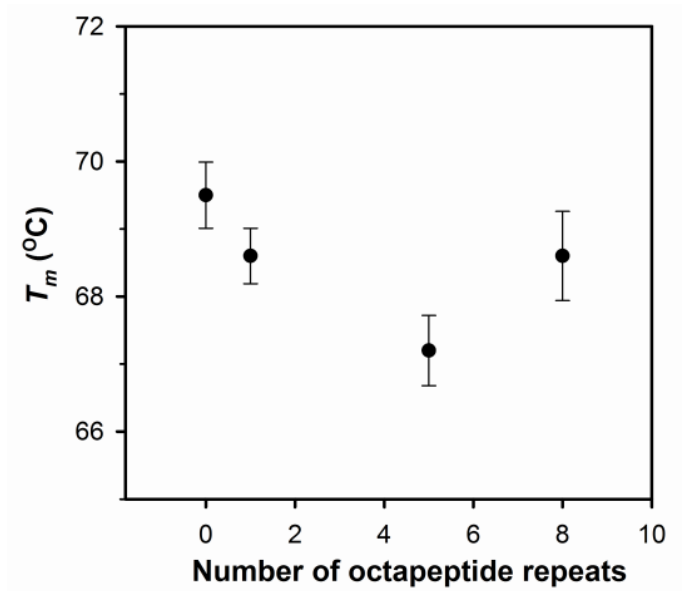

Figure 3. Thermal stability of four PrP variants compared by their $\mathrm{T}_{m}$ values.

\subsection{The Expansion Rather Than the Deletion of Octapeptides Enhances the Kinetics of Fibril Conversion}

Some selected data points in the kinetics of the fibril conversion are shown in Figure 4 and analyzed with a nucleation dependent polymerization model. Among four tested variants, PrP-8oct has the shortest lag time of $3.9 \mathrm{~h}$, while the PrP-1oct and PrP-5oct have their lag time of 5.0 and $4.8 \mathrm{~h}$, respectively. PrP- $\Delta$ oct has the longest lag time of $11.5 \mathrm{~h}$. If measuring the quantity of amyloid fibrils by maximum ThT fluorescence, converted fibrils are significantly higher in PrP-8oct and lower in PrP- $\Delta$ oct and PrP-1oct, in comparison to PrP-5oct. 


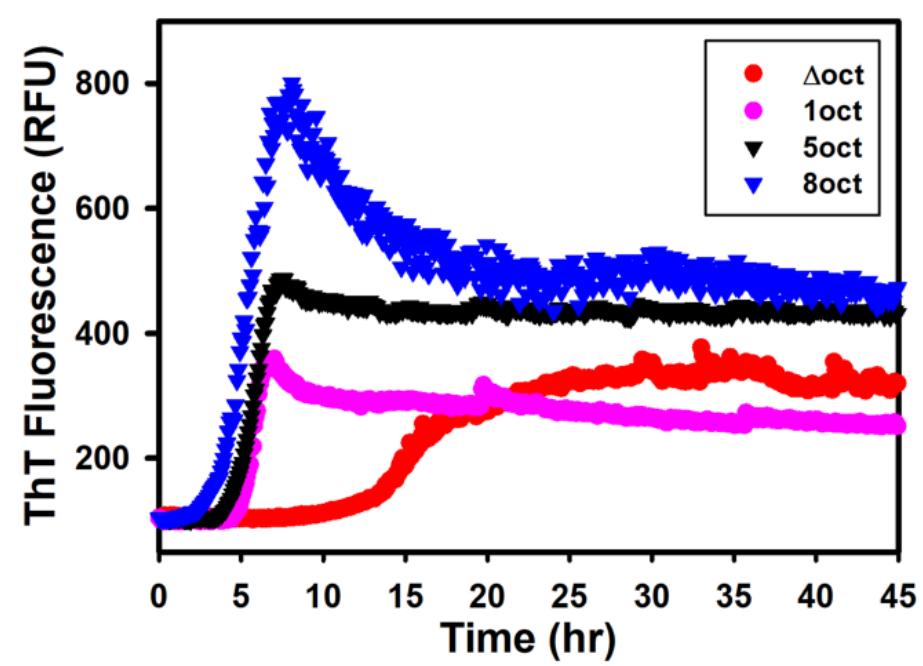

Figure 4. Kinetics of fibril conversion from four PrP variants.

\subsection{The Number of Octapeptide Repeats Affects the Structures of Fibrils}

The fibrils converted from PrP variants were analyzed for their secondary structure by $\mathrm{CD}$ spectroscopy (Figure $2 \mathrm{~b}$ ) and imaged with transmission electron microscopy (TEM) (Figure 5). After deletion or insertion of octapeptides, the fibrils have structures different from PrP-5oct. During the fibril conversion, PrP changes the structure from $\alpha$-helical structure into $\beta$-sheets but the level of the structural conversion is quite different among the four PrP variants. The levels of $\alpha$-helical structure represented by the negative peaks at $222 \mathrm{~nm}$ in four PrP variants were compared as shown in the insert (i). PrP-5oct preserves the least amount of $\alpha$-helical structure. Furthermore, to determine the contents of the structural change in each condition, the negative peaks representing $\beta$-sheet and $\alpha$-helical structures at $217 \mathrm{~nm}$ and $208 \mathrm{~nm}$, respectively, were put into ratios to characterize the helix-to-sheet structural change in fibril conversion. As shown in the inset (ii) of Figure $2 b$, PrP-5oct has the highest value of $[\theta] 217 /[\theta] 208$ at 1.83 and PrP-8oct has a value of 1.36 . In PrP- $\Delta$ oct and PrP-1oct, the values of $[\theta] 217 /[\theta] 208$ are only 1.20 and 1.32, respectively. The correlation of these ratios is similar to the number of octapeptide repeats vs. $\mathrm{T}_{m}$ (see Figure 3 and inset (ii) of Figure 2b). In other words, PrP-5oct has the weakest thermal stability and has the greatest structural change during fibril conversion.

As illustrated in Figure 5, PrP-5oct significantly turns into long silk-like fibrils and the fibrils populate evenly over the field. A high number of fibrils are observed in PrP-8oct as well. However, PrP-8oct fibrils are extremely fragmented along the long axis of their fibrils as compared to PrP-5oct fibrils. In addition, PrP-8oct fibrils aggregate, consistent with the decrease of ThT fluorescence after reaching the maximum reading in Figure 4. No oligomers are observed in the PrP-5oct and PrP-8oct fibril samples. In contrast to the long fibrils in PrP-5oct and PrP-8oct, PrP- $\triangle$ oct has very few fibrils formed and has many loosely formed oligomers present. The PrP-1oct has very few mature fibrils but retains moderate ThT-fluorescence as shown in Figure 4 . Similar to PrP- $\Delta$ oct, some protofibrils are present in the PrP-1oct fibril sample and these protofibrils seem to be more well-formed then those oligomers in PrP- $\Delta$ oct. Among the limited amount of PrP- $\Delta$ oct and PrP-1oct fibrils, a twisted form of fibrils is also identified. This twisted form is highly distinguishable from the silk-like form of PrP-5oct fibrils and the fragmented form of PrP-8oct fibrils. Overall, the population of these four fibril samples correlates with the observation that increasing the number of octapeptide repeats causes accumulation of large plaques [14]. 


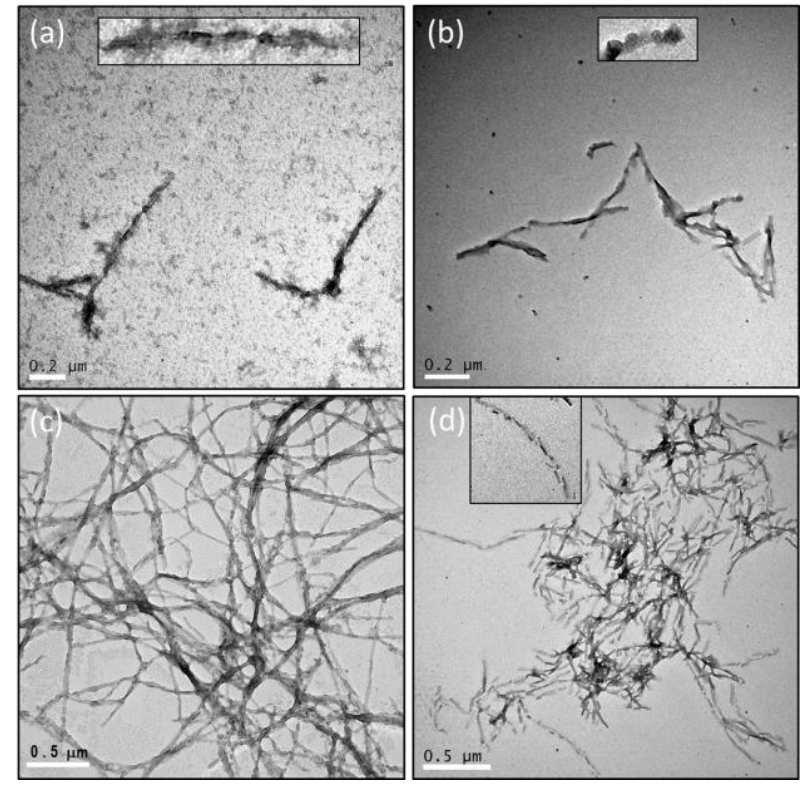

Figure 5. Transmission electron microscopy (TEM) images of fibrils converted from four PrP variants (a) PrP- $\Delta$ oct, (b) PrP-1oct, (c) PrP-5oct and (d) PrP-8oct.

A previous study on the correlation between structural stability and the incubation period indicated that less stable prions replicate more rapidly than stable prions and that the less stable prions quickly replicate during a fast breakup [15]. As aforementioned, PrP-5oct has the least stable structure, thus, it has the highest yield of fibril formation. The formation of fibril seeds is difficult when the fibrils cannot be easily fragmented, such as the twisted fibrils of PrP- $\Delta$ oct and PrP-1oct and typical silk-like fibrils of PrP-5oct as observed in many other amyloid proteins [16]. Considering the weak $\alpha$-helical structure and fragmented fibrils observed in PrP-8oct, it is highly possible that PrP-8oct is highly flexible to carry out structural change to form amyloid fibrils. In addition, these PrP-8oct fibrils easily fragment to serve as seeds for elongation of other misfolded PrP-8oct monomers. With abundant fibril seeds, PrP-8oct starts elongation in a short time. The density of fibrils shown in TEM images is very high in PrP-5oct and PrP-8oct but is very low in PrP- $\triangle$ oct and PrP-1oct. The ThT-fluorescence readings of PrP- $\Delta$ oct and PrP-1oct fibrils are approximately half of the ThT-fluorescence reading of PrP-5oct fibrils. The major reason is that the fibrils with different structures carry different levels of ThT-fluorescence [17]. The fibrils cannot be precisely quantified simply based on ThT-fluorescence of the four PrP variants because fibrils converted from these four PrP variants have different misfolding pathways resulting in distinguishable structures.

PK-resistance is a unique property of $\mathrm{PrP}^{\mathrm{Sc}}$ and a PK-digestion assay is one of the best ways to distinguish $\operatorname{PrP}^{\mathrm{C}}$ and $\operatorname{PrP} \mathrm{P}^{\mathrm{Sc}}$. Thus, we conducted PK-digestion assays on four $\operatorname{PrP}$ variants in both protein and fibril forms as illustrated in Figure 6. In their protein forms, PrP- $\triangle$ oct, PrP-1oct and PrP-5oct are all PK-sensitive, while PrP-8oct (25 kDa) is partially resistant to $P K$ as shown by the residual PrP band and small protein bands between 10 and $15 \mathrm{kDa}$. The PK-resistant protein fragments in PrP-8oct are critical as these octapeptide insertions are toxic before fibril conversion is carried out. In their fibril forms, all PrP variants have PK-resistant bands observed at $\sim 10 \mathrm{kDa}$, regardless of the structures of these fibrils. Consistent with a study of cell-expressed hamster PrP, PK-resistance is significantly increased upon octapeptide insertion [18]. 

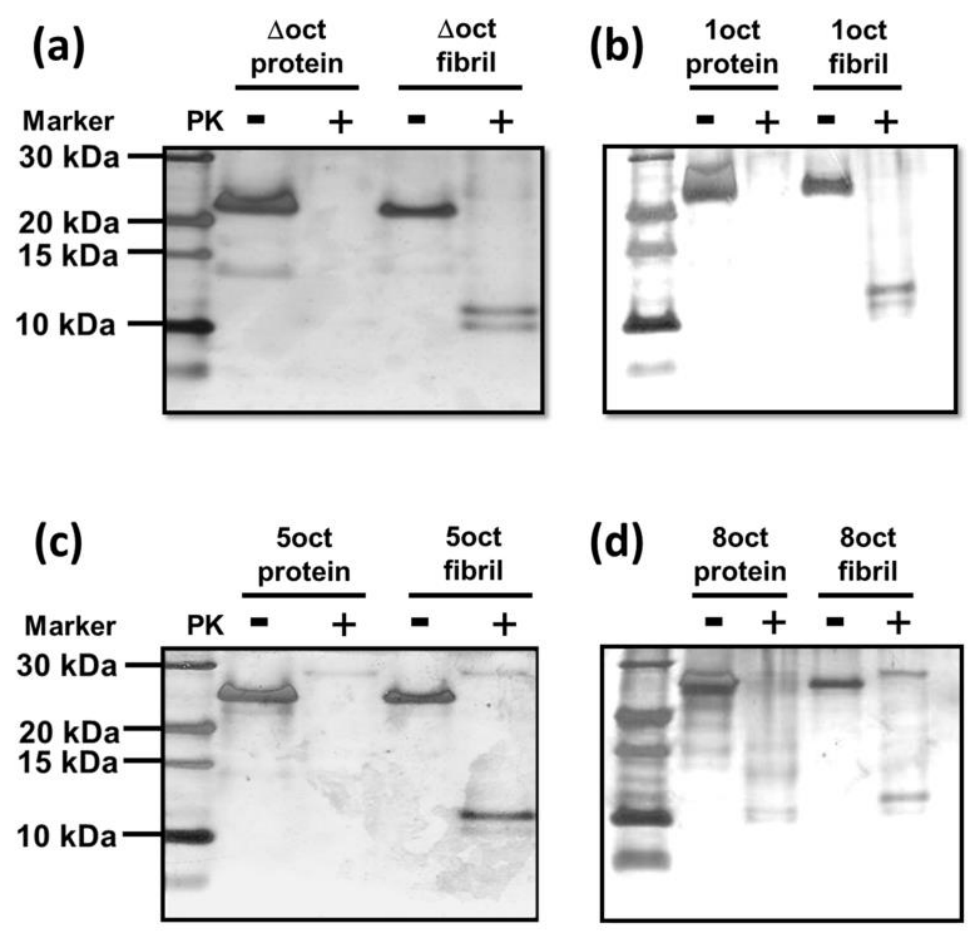

Figure 6. PK-cleavage ( $\mathrm{PK}: \operatorname{PrP}=1: 50)$ of PrP variants (a) PrP- $\Delta$ oct, (b) PrP-1oct, (c) PrP-5oct and (d) PrP-8oct in their protein and fibril forms analyzed with tricine-sodium dodecyl sulfatepolyacrylamide gel electrophoresis (SDS-PAGE).

\subsection{The Number of Octapeptide Repeats Affects the Cytotoxicity of the Amyloid Fibrils}

After the characterization of the amyloid fibrils in a cell-free system, we further examined the toxicity of these fibrils in neuron-like differentiated mouse neuroblastoma (N2a) cells by cell viability assay as shown in Figure 7. In comparison to the untreated N2a cells, cell viability decreases with the number of octapeptide repeats of the fibril samples. Fibrils converted from $\operatorname{PrP}$ with a smaller number of octapeptide repeats contribute lower cytotoxicity but insertion of octapeptides (PrP-8oct) does not increase cytotoxicity in comparison to PrP-5oct. In our PrP- $\triangle$ oct fibril sample, lots of loosely formed oligomers are observed but they affect cell viability weakly. PrP-1oct fibrils have similar structures to PrP- $\Delta$ oct fibrils but PrP-1oct fibrils are more cytotoxic than PrP- $\Delta$ oct fibrils. This cytotoxicity difference could be due to the presence of protofibrils rather than oligomers in the PrP-1oct fibril sample. PrP-5oct and PrP-8oct fibrils have distinct structures but their cytotoxicity is independent of their fibril structures, although the fragmentation of PrP-8oct fibrils produce some short fibrils. There might be some threshold effect and increasing the number of peptides beyond a certain limit has no further effect on the cell viability, though the reasons are unclear at this stage. Considering that PrP-8oct converts into fibrils quickly as it causes damage earlier than PrP-5oct does, it is correlated with the early onset of diseases observed clinically [13]. There are fewer cases of patients with prion diseases [14] and weak cytotoxicity of PrP- $\Delta$ oct and PrP-1oct correlates with fewer cases of patients with prion diseases. 


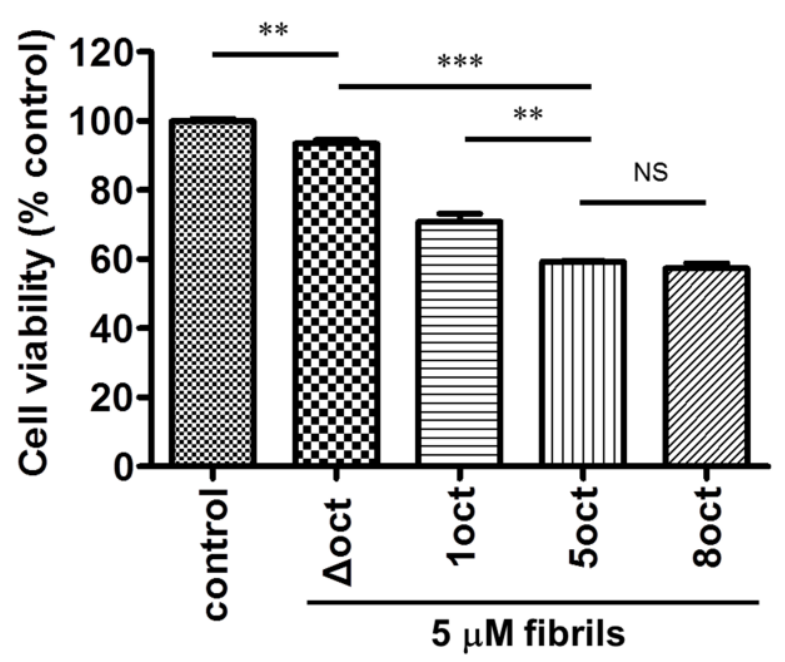

Figure 7. Cell viability of N2a cells upon treatment with fibrils converted from four PrP variants. The control sample is N2a cells without fibril treatment. ${ }^{* *}: p<0.01,{ }^{* * *}: p<0.001$, NS: non-significant.

\section{Discussion}

Extensive studies of recombinant prion proteins indicate that $\mathrm{PrP}_{90-231}$ can potentially convert to amyloid fibrils with PK-resistance [19] and are encephalopathological to mice [20]. Extending the N-terminal sequence to full-length $\operatorname{PrP}, \operatorname{PrP}_{23-231}$ tends to produce similar amyloid fibrils with amyloidogenic cores in the C-terminal domain [21]. An amyloidogenic core has been determined to be $\mathrm{PrP}_{121-231}$, when fibrillization of $\mathrm{PrP}_{121-231}$ was reported [9]. The octapeptides are not located in the amyloidogenic core, thus, the critical role of octapeptides in the flexible N-terminal domain has been strongly underestimated.

Our CD spectra indicate that insertion and deletion of octapeptides change the fraction of $\alpha$-helical structure. This result indicates that the $\mathrm{N}$-terminal domain has some influence over the globular structure in the C-terminal domain. Our CD result is consistent with a previous study that showed truncation of the N-terminal domain causes a shift in the NMR spectrum [22]. The N-terminal domain has an extremely high level of disorder and this property decreases significantly when extending the protein sequence to the globular C-terminal domain. Helix I (equal to $\alpha 1$ in Figure 1) has been considered a highly mobile segment from the amyloid core [23,24]. Changing the number of octapeptide repeats initiates the weakening of the helical structures and this flexibility promotes its role as a hydrophilic seed in prion aggregates [25]. As a result, $\mathrm{PrP}$ with multiple octapeptide repeats has a higher tendency to weaken the rigid $\alpha$-helical structure when carrying out structural conversion. In contrast, $\operatorname{PrP}$ with a lower number of octapeptide repeats tends not to weaken $\alpha$-helical structure as much. Therefore, PrP-5oct and PrP-8oct have similar structures, whereas PrP- $\Delta$ oct and PrP-1oct have distinct structural differences from PrP-5oct.

Two types of $\alpha$-synuclein fibrils converted under identical conditions have been identified [26]. An animal study of $\alpha$-synuclein fibrils with distinct structure indicated that structure might determine the toxicity of the fibrils [27]. Similarly, two distinct structures of PrP fibrils converted from the same protein produced different levels of cytotoxicity [28]. Huntington's disease has also been ascribed to misfolding of huntingtin protein caused by an increase of polyglutamine [29]. Similarly, insertion of octapeptides is correlated with the early onset of CJD $[13,14]$. Our study indicates that insertion of octapeptides weakens the $\alpha$-helical structure, accelerates prion fibrillation and causes significant changes in fibril structure. The deletion of octapeptides strengthens the $\alpha$-helical structure. PrP variants with different structures then misfold into different amyloid structures. Immunocytochemical examination on patients indicated that the number of octapeptide repeats determines the type of amyloid deposit in the cerebellum and the corresponding prion encephalopathy [30]. As the prion proteins carrying different numbers of octapeptide repeats convert 
to distinct structures of fibrils, these fibrils could possibly deposit in different parts of the cerebellum and reflect the severity of the diseases.

\section{Materials and Methods}

\subsection{Plasmid Preparations of PrP- $\Delta$ oct, PrP-1oct and PrP-8oct}

Two oligonucleotides for PrP- $\triangle$ oct were used in this reaction including a forward primer (5'-TATCCCGGGCAGGGAAGCCCTGGAGGCAACCGTTACCCA- $\left.{ }^{\prime}\right)$ and a reverse primer (5'-TGGGTACCCCCTCCTGGGTAACGGTTGCCTCCAG-3'). In a template-repeated polymerase chain reaction (TR-PCR) [31], the two primers were also used as templates.

Similarly, PrP-1oct plasmid was prepared based on TR-PCR from the following primers: a forward primer (5'-TATCCCGGGCAGGGAAGCCCTGGAGGCAAC CGTTACC CACCCCACGGTGG-3') and a reverse primer (5'-ATGGGTACCC CCTCCTTGTCCCCAG CCACCACCGTGGGGTGGGTAA-3').

The wild type mouse PrP (PrP-5oct) gene has been constructed previously [32]. To delete five octapeptide repeats in PrP-5oct, a SmaI and a KpnI cleavage sites were used to remove all octapeptides in mouse PrP-5oct. After the digestion with restriction enzymes, the TR-PCR products were subcloned into pET101/D-TOPO vectors. To insert additional copies of the octapeptide into PrP-5oct, we first conducted site-directed mutagenesis to change the sequence at codons 54 and 55 of PrP-5oct to create a BamHI cleavage site. After digestion with restriction enzymes Bsu36I and BamHI, additional oligonucleotides for insertion of three octapeptide repeats were subcloned into PrP-5oct resulting in PrP-8oct. The inserted oligonucleotides are as following. The forward primer: TCAGGGTGGCACCTGGGGGCAGCCCCACGGTGGTGGCTGGGGACAACCACATGGTGGTTCTTGGGGTCAACCTCACGGTG. The reverse primer: GATCCACCGTGAGGTTGACCCCAAGAACCACCATGTGGTTGTCCCCAGCCACCACCGTGGGGCTG CCCCCAGGTGCCACCC. To be noted, this engineering introduces a Thr to Ser change at codon 55 in PrP-5oct but this Ser becomes part of R3 that follows the inserted sequence as shown in Figure 1. Therefore, except for the insertion, the wild type sequence is not changed.

\subsection{Expression and Purification of PrP- $\triangle$ oct, PrP-1oct, PrP-5oct and PrP-8oct}

The plasmids pET101 encoding PrP- $\Delta$ oct, PrP-1oct, PrP-5oct and PrP-8oct were transformed into competent Escherichia coli BL21 star ${ }^{\mathrm{TM}}$ (DE3) (Life Technologies, Grand Island, NY, USA) cells, in $50 \mathrm{~mL}$ of LB medium for approximately 16 hours. From a large-scale culture, cells were transferred to a TB medium and grown until an optical density at $600 \mathrm{~nm}$ reached 0.6. Subsequent addition of $1 \mathrm{mM}$ isopropyl $\beta$-D-1-thiogalactopyranoside induced expression of proteins. The PrP-5oct and PrP-8oct proteins were purified by immobilized metal affinity chromatography and reversed-phase C4-HPLC as modified from previously reported procedures [32-34]. The PrP- $\Delta$ oct and PrP-1oct proteins were purified by cation exchange chromatography with SP Sepharose and reversed-phase C4-HPLC as previously described [35]. The purified PrP was confirmed by sodium dodecyl sulfate-polyacrylamide gel electrophoresis (SDS-PAGE) and electrospray ionization-mass spectrometry to be a single population with the correct molecular weight.

\subsection{Circular Dichroism Spectroscopy (CD)}

CD spectra of mouse PrP variants were recorded with a spectrometer (Jasco J-815, Tokyo, Japan). For measurements in the far-UV region, a quartz cell with a path length of $0.1 \mathrm{~cm}$ was used in a nitrogen atmosphere. For protein samples, the concentration was kept constant at $10 \mu \mathrm{M}$ in $10 \mathrm{mM}$ Tris (pH 7.3). For fibril samples, the samples were dialyzed against water prior to the measurements. A set of five scans, with a scan speed of $50 \mathrm{~nm}$ per minute, was performed at $20^{\circ} \mathrm{C}$. The fraction of the secondary structures was analyzed by DichroWeb [36,37].

The heat-induced denaturation of proteins was conducted with heating protein solutions at the rate of $1{ }^{\circ} \mathrm{C} / \mathrm{min}$ and the ellipticity at $222 \mathrm{~nm}$, was recorded every $0.5{ }^{\circ} \mathrm{C}$. The 
thermal stability of proteins was analyzed based on the Gibbs-Helmholtz equation and the melting temperatures $\left(\mathrm{T}_{m}\right)$ of mouse $\operatorname{PrP}$ variants were determined.

\subsection{Kinetics of Fibril Conversion from PrP Variants}

For fibril conversion, four PrP proteins were added into $50 \mathrm{mM}$ MES (pH 6) in the presence of $2 \mathrm{M}$ guanidine hydrochloride $(\mathrm{GdnHCl})$. The samples were incubated at $37^{\circ} \mathrm{C}$ with vigorous shaking, as described in previous studies on prion fibrillation $[34,35,38]$. At the end of the experiment, the fibril samples were dialyzed against water for further analysis. For the kinetics experiment, the fibril conversion was carried out in a multiwell plate rotating at $600 \mathrm{rpm}$ at $37{ }^{\circ} \mathrm{C}$ monitored by the fluorescence of thioflavin T (ThT) in a microplate reader (FLUOstar Omega, BMG LABTECH, Ortenberg, Germany). The kinetics of fibril conversion were analyzed by the nucleation dependent polymerization model [22]. The lag phase of amyloid formation from $\mathrm{PrP}$ can be determined by fitting the time-dependent changes in the ThT fluorescence $(\mathrm{F})$ over time $(\mathrm{t})$ of the reaction as expressed in the following equation, $\mathrm{F}=\mathrm{F}_{0}+\Delta \mathrm{F} /\left(1+\exp \left[k\left(t_{m}-t\right)\right]\right)$. Where $\mathrm{F}_{0}$ is the minimum level of ThT fluorescence during the lag phase, $\Delta \mathrm{F}$ is the difference of ThT fluorescence between the maximum level (steady state) and the minimum level (lag phase), $k$ is the fibril growth rate $\left(\mathrm{h}^{-1}\right)$ and $t_{m}$ is the observed time at the midpoint of transition. The lag time $\left(t_{l}\right)$ of fibril formation can be calculated as: $t_{l}=t_{m}-2 / k$.

\subsection{Transmissible Electron Microscopy (TEM)}

The diluted fibril samples were adsorbed onto carbon-coated 200-mesh copper grids for $30 \mathrm{~s}$ and washed twice with PBS buffer. Subsequently, the adsorbed fibrils were stained with $2 \%$ tungsten phosphoric acid on copper grids and then washed with $\mathrm{H}_{2} \mathrm{O}$. The samples were air-dried before imaging. The TEM images were collected using a TEM (Hitachi H-7100, Tokyo, Japan).

\subsection{PK-Digestion Assay}

In this assay, $10 \mu \mathrm{M}$ of protein and fibril samples were treated with PK $(29 \mathrm{kDa})$ at a PrP-to-PK ratio of $1: 50$ at $37^{\circ} \mathrm{C}$ in $100 \mathrm{mM}$ Tris (pH 7.5) for one hour. After PK-digestion, samples were analyzed by tricine-SDS-PAGE [39].

\subsection{Cell Culture and Viability Assay}

N2a cells were maintained in Dulbecco's modified Eagle's medium (DMEM) supplemented with penicillin/streptomycin and 10\% $(v / v)$ fetal bovine serum in a humidified atmosphere with $5 \% \mathrm{CO}_{2}$ at $37^{\circ} \mathrm{C}$. In the following incubation, N2a cells $\left(1 \times 10^{4}\right)$ were seeded to 96-well plates for $24 \mathrm{~h}$ in a serum-free DMEM medium to differentiate into a neuron-like morphology and then treated with PrP fibrils. After $72 \mathrm{~h}$ of incubation, the medium was removed and the cell plates were washed by PBS. Subsequently, 10\% (v/v) WST-1 was added into the plates and incubated with cells for $3 \mathrm{~h}$ in the dark. During the incubation, the cleavage of the tetrazolium salt WST-1 to formazan by cellular mitochondrial dehydrogenases was carried out. The cell viability was determined by the absorption of formazan recorded at $450 \mathrm{~nm}$ using an ELISA reader. The collected data were compared using $T$-test statistical analysis.

\section{Conclusions}

Increasing octapeptide repeats accelerates prion fibrillation and induces significant change in fibril structure, whereas decreasing octapeptide repeats enhances the intramolecular $\alpha$-helical structures but forms fewer twisted fibrils. Increasing the number of octapeptide repeats turns PrP into partially PK-resistant before fibril conversion. Deletion of octapeptides weakens the cytotoxicity but insertion of octapeptides does not increase the cytotoxicity. The effect of octapeptide repeats observed in this study explains the molecular mechanism of octapeptide repeats at the onset of prion diseases. Prior to this research, the 
octapeptides were not considered as important in prion diseases. Our study indicates that the octapeptides are critical in folding and misfolding of prion proteins.

Author Contributions: Conceptualization, C.-I.L.; investigation, K.-H.Y. and M.-Y.H.; resources, C.-I.L. and H.-R.C.; data curation, M.-Y.H., Y.-R.L. and Y.-K.L.; writing-original draft preparation, C.-I.L.; writing-review and editing, K.-H.Y. and C.-I.L.; supervision, C.-I.L.; project administration, C.-I.L.; funding acquisition, C.-I.L. All authors have read and agreed to the published version of the manuscript.

Funding: This research was funded by Ministry of Science and Technology under Grant MOST 104-2113-M-194-006, MOST 105-2113-M-194-005 and MOST 106-2113-194-009.

Institutional Review Board Statement: Not applicable.

Informed Consent Statement: Not applicable.

Data Availability Statement: Not applicable.

Acknowledgments: The authors thank Cheng-Liang Huang in National Chia-Yi University for technical support in TEM imaging.

Conflicts of Interest: The authors declare no conflict of interest.

\section{References}

1. Griffith, J.S. Self-replication and scrapie. Nature 1967, 215, 1043-1044. [CrossRef]

2. Prusiner, S.B. Novel proteinaceous infectious particles cause scrapie. Science 1982, 216, 136-144. [CrossRef]

3. Prusiner, S.B. Prion biology and diseases-Laughing cannibals, mad cows, and scientific heresy. Med. Res. Rev. 1996, 16, 487-505. [CrossRef]

4. Palmer, M.S.; Mahal, S.P.; Campbell, T.A.; Hill, A.F.; Sidle, K.C.; Laplanche, J.-L.; Collinge, J. Deletions in the prion protein gene are not associated with CJD. Hum. Mol. Genet. 1993, 2, 541-544. [CrossRef]

5. Reder, A.T.; Mednick, A.S.; Brown, P.; Spire, J.P.; Van Cauter, E.; Wollmann, R.L.; Cervenakova, L.; Goldfarb, L.G.; Garay, A.; Ovsiew, F.; et al. Clinical and genetic studies of fatal familial insomnia. Neurology 1995, 45, 1068-1075. [CrossRef]

6. Perry, R.T.; Go, R.C.; Harrell, L.E.; Acton, R.T. SSCP analysis and sequencing of the human prion protein gene (PRNP) detects two different 24 bp deletions in an atypical Alzheimer's disease family. Am. J. Med. Genet. 1995, 60, 12-18. [CrossRef]

7. Zahn, R.; Von Schroetter, C.; Wüthrich, K. Human prion proteins expressed in Escherichia coli and purified by high-affinity column refolding. FEBS Lett. 1997, 417, 400-404. [CrossRef]

8. Pan, K.M.; Baldwin, M.; Nguyen, J.; Gasset, M.; Serban, A.; Groth, D.; Mehlhorn, I.; Huang, Z.; Fletterick, R.J.; Cohen, F.E.; et al. Conversion of $\alpha$-helices into $\beta$-sheets features in the formation of the scrapie prion proteins. Proc. Natl. Acad. Sci. USA 1993, 90, 10962-10966. [CrossRef]

9. Martins, S.M.; Frosoni, D.J.; Martinez, A.M.B.; De Felice, F.G.; Ferreira, S.T. Formation of soluble oligomers and amyloid fibrils with physical properties of the scrapie isoform of the prion protein from the C-terminal domain of recombinant murine prion protein mPrP-(121-231). J. Biol. Chem. 2006, 281, 26121-26128. [CrossRef] [PubMed]

10. Millhauser, G.L. Copper binding in the prion protein. Acc. Chem. Res. 2004, 37, 79-85. [CrossRef] [PubMed]

11. Whittal, R.M.; Ball, H.L.; Cohen, F.E.; Burlingame, A.L.; Prusiner, S.B.; Baldwin, M.A. Copper binding to octarepeat peptides of the prion protein monitored by mass spectrometry. Protein Sci. 2000, 9, 332-343. [CrossRef] [PubMed]

12. Wopfner, F.; Weidenhöfer, G.; Schneider, R.; Von Brunn, A.; Gilch, S.; Schwarz, T.F.; Werner, T.; Schätzl, H.M. Analysis of 27 mammalian and 9 avian PrPs reveals high conservation of flexible regions of the prion protein. J. Mol. Biol. 1999, 289, 1163-1178. [CrossRef]

13. Croes, E.; Theuns, J.; Houwing-Duistermaat, J.; Dermaut, B.; Sleegers, K.; Roks, G.; Broeck, M.V.D.; Van Harten, B.; Van Swieten, J.C.; Cruts, M.; et al. Octapeptide repeat insertions in the prion protein gene and early onset dementi. J. Neurol. Neurosurg. Psychiatry 2004, 75, 1166-1170. [CrossRef] [PubMed]

14. Beck, J.; Poulter, M.; Campbell, T.A.; Adamson, G.; Uphill, J.B.; Guerreiro, R.; Jackson, G.S.; Stevens, J.C.; Manji, H.; Collinge, J.; et al. PRNP allelic series from 19 years of prion protein gene sequencing at the MRC Prion Unit. Hum. Mutat. 2010, 31, E1551-E1563. [CrossRef]

15. Legname, G.; Nguyen, H.-O.B.; Peretz, D.; Cohen, F.E.; DeArmond, S.J.; Prusiner, S.B. Continuum of prion protein structures enciphers a multitude of prion isolate-specified phenotypes. Proc. Natl. Acad. Sci. USA 2006, 103, 19105-19110. [CrossRef] [PubMed]

16. Caughey, B.; Lansbury, P.T. Protofibrils, pores, fibrils, and neurodegeneration: Separating the responsible protein aggregates from the innocent bystanders. Annu. Rev. Neurosci. 2003, 26, 267-298. [CrossRef]

17. Makarava, N.; Baskakov, I.V. The same primary structure of the prion protein yields two distinct self-propagating states. J. Biol. Chem. 2008, 283, 15988-15996. [CrossRef] [PubMed] 
18. Priola, S.A.; Chesebro, B. Abnormal properties of prion protein with insertional mutations in different cell types. J. Biol. Chem. 1998, 273, 11980-11985. [CrossRef]

19. Swietnicki, W.; Morillas, M.; Chen, S.G.; Gambetti, P.; Surewicz, W.K. Aggregation and fibrillization of the recombinant human prion protein huPrP90-231. Biochemistry 2000, 39, 424-431. [CrossRef]

20. Legname, G.; Baskakov, I.V.; Nguyen, H.-O.B.; Riesner, D.; Cohen, F.E.; DeArmond, S.J.; Prusiner, S.B. Synthetic mammalian prions. Science 2004, 305, 673-676. [CrossRef] [PubMed]

21. Bocharova, O.V.; Breydo, L.; Salnikov, V.V.; Gill, A.C.; Baskakov, I.V. Synthetic prions generated in vitro are similar to a newly identified subpopulation of $\mathrm{PrP}^{\mathrm{Sc}}$ from sporadic Creutzfeldt-Jakob disease. Protein Sci. 2005, 14, 1222-1232. [CrossRef]

22. Leliveld, S.R.; Stitz, L.; Korth, C. Expansion of the octarepeat domain alters the misfolding pathway but not the folding pathway of the prion protein. Biochemistry 2008, 47,6267-6278. [CrossRef]

23. De Simone, A.; Zagari, A.; Derreumaux, P. Structural and hydration properties of the partially unfolded states of the prion protein. Biophys. J. 2007, 93, 1284-1292. [CrossRef]

24. Viles, J.H.; Donne, D.; Kroon, G.; Prusiner, S.B.; Cohen, F.E.; Dyson, H.J.; Wright, P.E. Local structural plasticity of the prion protein. Analysis of NMR relaxation dynamics. Biochemistry 2001, 40, 2743-2753. [CrossRef]

25. Morrissey, M.P.; Shakhnovich, E.I. Evidence for the role of $\mathrm{PrP}^{\mathrm{C}}$ helix 1 in the hydrophilic seeding of prion aggregates. Proc. Natl. Acad. Sci. USA 1999, 96, 11293-11298. [CrossRef] [PubMed]

26. Heise, H.; Hoyer, W.; Becker, S.; Andronesi, O.C.; Riedel, D.; Baldus, M. Molecular-level secondary structure, polymorphism, and dynamics of full-length alpha-synuclein fibrils studied by solid-state NMR. Proc. Natl. Acad. Sci. USA 2005, 102, 15871-15876. [CrossRef] [PubMed]

27. Peelaerts, W.; Bousset, L.; Van Der Perren, A.; Moskalyuk, A.; Pulizzi, R.; Giugliano, M.; Haute, C.V.D.; Melki, R.; Baekelandt, V. alpha-Synuclein strains cause distinct synucleinopathies after local and systemic administration. Nature 2005, 522, 340-344. [CrossRef] [PubMed]

28. Lee, Y.J.; Savtchenko, R.; Ostapchenko, V.G.; Makarava, N.; Baskakov, I.V. Molecular structure of amyloid fibrils controls the relationship between fibrillar size and toxicity. PLoS ONE 2011, 6, e20244. [CrossRef]

29. Scherzinger, E.; Lurz, R.; Turmaine, M.; Mangiarini, L.; Hollenbach, B.; Hasenbank, R.; Bates, G.P.; Davies, S.W.; Lehrach, H.; E Wanker, E. Huntingtin-encoded polyglutamine expansions form amyloid-like protein aggregates in vitro and in vivo. Cell 1997, 90, 549-558. [CrossRef]

30. Vital, C.; Gray, F.; Vital, A.; Parchi, P.; Capellari, S.; Petersen, R.B.; Ferrer, X.; Jarnier, D.; Julien, J.; Gambetti, P. Prion encephalopathy with insertion of octapeptide repeats: The number of repeats determines the type of cerebellar deposits. Neuropathol. Appl. Neurobiol. 1998, 24, 125-130. [CrossRef]

31. Hsu, C.T.; Ting, C.Y.; Ting, C.J.; Chen, T.Y.; Lin, C.P.; Whang-Peng, J.; Hwang, J. Vaccination against gonadotropin-releasing hormone (GnRH) using toxin receptor-binding domain-conjugated GnRH repeats. Cancer Res. 2000, 60, 3701-3705.

32. Bocharova, O.V.; Breydo, L.; Parfenov, A.S.; Salnikov, V.V.; Baskakov, I.V. In vitro conversion of full-length mammalian prion protein produces amyloid form with physical properties of PrPSc. J. Mol. Biol. 2005, 346, 645-659. [CrossRef]

33. Luo, J.-C.; Wang, S.-C.; Jian, W.-B.; Chen, C.-H.; Tang, J.-L.; Lee, C.-I. Formation of amyloid fibrils from $\beta$-amylase. FEBS Lett. 2012, 586, 680-685. [CrossRef] [PubMed]

34. Lin, S.-J.; Yu, K.-H.; Wu, J.-R.; Lee, C.-F.; Jheng, C.-P.; Chen, H.-R.; Lee, C.-I. Liberation of GPI-anchored prion from phospholipids accelerates amyloidogenic conversion. Int. J. Mol. Sci. 2013, 14, 17943-17957. [CrossRef] [PubMed]

35. Jen, H.-I.; Lin, Z.-Y.; Guo, J.-X.; Lee, C.-I. The effects of divalent cation-chelated prion fibrils on the immune response of EOC 13.31 microglia cells. Cells 2020, 9, 2285. [CrossRef] [PubMed]

36. Whitmore, L.; Wallace, B.A. Protein secondary structure analyses from circular dichroism spectroscopy: Methods and reference databases. Biopolymers 2007, 89, 392-400. [CrossRef] [PubMed]

37. Whitmore, L.; Wallace, B.A. DICHROWEB, an online server for protein secondary structure analyses from circular dichroism spectroscopic data. Nucleic Acids Res. 2004, 32, W668-W673. [CrossRef]

38. Makarava, N.; Lee, C.-I.; Ostapchenko, V.G.; Baskakov, I.V. Highly promiscuous nature of prion polymerization. J. Biol. Chem. 2007, 282, 36704-36713. [CrossRef]

39. Schagger, H. Tricine-SDS-PAGE. Nat. Protoc. 2006, 1, 16-22. [CrossRef] 